\title{
Immobilized enzyme-single-wall carbon nanotube composites for amperometric glucose detection at a very low applied potential $\dagger$
}

\author{
Michael E. G. Lyons* and Gareth P. Keeley \\ Received (in Cambridge, UK) 6th December 2007, Accepted 4th March 2008 \\ First published as an Advance Article on the web 28th March 2008 \\ DOI: $10.1039 / b 718863 c$
}

\begin{abstract}
The behaviour of support electrodes modified with randomly dispersed single-wall carbon nanotube meshes containing adsorbed glucose oxidase with respect to amperometric glucose detection at a low potential is demonstrated.
\end{abstract}

Electron transfer in biological systems is one of the leading areas in the biochemical and biophysical sciences, ${ }^{1}$ and in recent years there has been considerable interest in the direct electron transfer between redox proteins and electrode surfaces. ${ }^{2}$ However in the absence of mediating small molecules, the observation of well defined electrochemical behaviour of immobilized flavoprotein oxidase systems such as Glucose Oxidase (GOx) is rendered extremely difficult, because the FAD group is embedded deep within the protein structure thereby making the transmission coefficient for direct electron transfer between the latter and a support electrode very small. ${ }^{3}$ Various immobilization strategies ${ }^{4,5}$ have been adopted to fabricate enzyme electrodes for biosensor applications. These strategies have exhibited variable degrees of success and in many cases electron transfer mediators have been used to facilitate electronic communication between the active site of the protein and the underlying electrode. However, the potential at which an amperometric enzyme biosensor is operated depends on the redox potential of the mediator used rather than that exhibited by the active site of the redox enzyme. Usually the difference in magnitude between the latter potentials is significant (ca. 0.3-0.5 V) and is a factor which acts against successful biosensor operation, since the more positive the operating potential, the greater the tendency is for the sensor to respond to oxidizable substances present in the sample other than the target substrate. Clearly the best strategy for successful enzyme biosensor fabrication is to devise a configuration by which electrons can directly transfer from the redox center of the enzyme to the underlying support electrode. This has been accomplished in recent years using the concept of molecular wiring.

The similarity in length scales between carbon nanotubes and redox enzymes suggests the presence of interactions that may be favourable for biosensor application. ${ }^{6}$ The strategy of physical adsorption or covalent immobilization of large bio-

Physical and Materials Electrochemistry Laboratory, School of Chemistry, University of Dublin, Trinity College, Dublin 2, Ireland. E-mail:melyons@tcd.ie; Fax: +353-1-6712826,

Tel: $+353-1-8962051$

$\dagger$ Electronic supplementary information (ESI) available: Extended discussion of enzyme activity and nanotube catalytic mechanism. See DOI: $10.1039 / \mathrm{b} 718863 \mathrm{c}$ molecules onto the surface of immobilized carbon nanotubes may well represent an exciting pathway through which direct electrical communication between support electrodes and the active site of redox enzymes can be achieved. For instance recent work ${ }^{7,8}$ has indicated that the chemical modification of electrode surfaces with carbon nanotubes has enhanced the activity of the latter with respect to the reaction of biologically active species such as hydrogen peroxide, dopamine and NADH. Furthermore multi-walled carbon nanotubes have been shown to exhibit good electronic communication with redox proteins where not only the redox center is close to the proteins surface such as found with cytochrome c, azurin and horseradish peroxidase, but also when it is embedded deep with the glycoprotein sheath such as is found with glucose oxidase. ${ }^{9,10}$ In the present paper we report on the well resolved redox behaviour and excellent catalytic properties of glucose oxidase adsorbed on the surface of single wall carbon nanotubes which have been dispersed on the surface of support electrodes to form a random mesh of high surface area.

In our experiments a suspension of single-walled carbon nanotubes was prepared by adding $1 \mathrm{mg}$ SWCNTs (HIPCO type, Carbon Nanotechnologies Inc., Houston, Texas, typical Fe content $15 \%$ by mass) to dimethyl formamide $\left(10 \mathrm{~cm}^{3}\right)$ and sonicating for $5 \mathrm{~min}$. This suspension $(1 \mu \mathrm{L})$ was cast on inverted gold or glassy carbon electrodes and the DMF was evaporated using a fan heater at $40{ }^{\circ} \mathrm{C}$ for approximately $15 \mathrm{~min}$. The electrode was rinsed sequentially with water and supporting electrolyte solution before all experiments. A solution of glucose oxidase (GOx, Type VII from Aspergillus Niger, $198 \mathrm{U} \mathrm{mg}^{-1}$, Sigma) was prepared by adding GOx (3 $\mathrm{mg}$ ) to a $50 \mathrm{mM} \mathrm{pH} 7$ phosphate buffer solution $\left(1 \mathrm{~cm}^{3}\right)$. This solution $(10 \mu \mathrm{L})$ was deposited on a SWCNT modified electrode and the solvent allowed evaporate at room temperature for $c a$. 2.5 h. Finally, alcoholic Nafion solution $(1 \mu \mathrm{L}, 5 \%$ $\mathrm{w} / \mathrm{v}$, Sigma) was cast onto the modified electrode surface and the solvent allowed to evaporate at room temperature. The resulting film covered the gold working electrode and the surrounding epoxy sheath and was clearly visible to the naked eye. This procedure resulted in the fabrication of a porous large surface area randomly dispersed SWCNT/GOx mesh on the electrode surface.

The redox behaviour of the SWCNT/GOx/Nafion composite modified electrode was investigated using cyclic voltammetry and potential step chronoamperometry. The voltammograms presented in Fig. 1 were obtained at an unmodified, SWCNT-modified and SWCNT/GOx/Nafion modified gold electrodes in contact with glucose free 


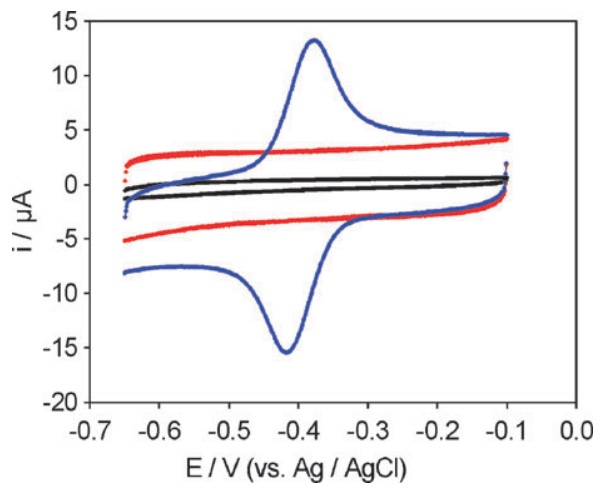

Fig. 1 Cyclic voltammograms recorded at a bare (black), SWCNT modified (red) and SWCNT/GOx/Nafion modified (blue) electrode at a sweep rate of $100 \mathrm{~V} \mathrm{~s}^{-1}$ in phosphate buffer solution $\mathrm{pH} 7$.

phosphate buffer solution (50 mM, pH 7). As can be seen, while an unmodified electrode exhibits a virtually featureless voltammetric response, and the SWCNT modified electrode exhibits a distinct capacitative response, a well defined pair of redox peaks was observed for the immobilized enzyme electrode. The latter are characteristic of the $\mathrm{FAD} / \mathrm{FADH}_{2}$ redox couple. Pertinent data extracted from the voltammogram at a sweep rate of $100 \mathrm{mV} \mathrm{s}^{-1}$ include $E^{0}=-396 \mathrm{mV}$, peak separation $\Delta E=41 \mathrm{mV}$, surface coverage $\Gamma=1.7 \mathrm{nmol}$ $\mathrm{cm}^{-2}$. The excellent peak definition observed is noteworthy and may be attributed to the fact that SWCNT are readily solubilized in Nafion solutions made from alcoholic components. ${ }^{11}$ The structure of Nafion in solution may be viewed as a fluorocarbon backbone with protruding polar sulfonate $\mathrm{SO}_{3}{ }^{-}$ groups. Hence it is similar in nature to other polymers which have been used to wrap and solubilize CNT material. The wrapping of nanotubes by water soluble polymers such as Nafion $^{12}$ is a general phenomenon driven by a thermodynamic impetus to eliminate the hydrophobic interface between the tubes and the aqueous medium, thereby reducing the density of the tangled tube assembly on the electrode surface. This may well increase the permeability of the void space between the tubes to increased quantities of electrolyte ions which will result in a change in the interfacial potential distribution and hence on the voltammetric response. ${ }^{13}$ Ideally $\Delta E$ should be zero for surface immobilized redox systems. ${ }^{14}$ The non zero value obtained in our experiments may be attributed to the presence of a potential difference between the support electrode and the site of electron transfer (the flavin group). ${ }^{15}$ Hence the presence of Nafion while enhancing the discrimination between the Faradaic and Capacitative current contributions associated with the immobilized flavin group, may well result in a large potential drop in the interface region. We have recently shown ${ }^{16}$ that the $\mathrm{FAD} / \mathrm{FADH}_{2}$ redox kinetics of this system may be directly probed via potential step chronoamperometry. Deviations from the expected 1st order kinetic response were observed indicating the presence of kinetic dispersion. Application ${ }^{16}$ of the Gaussian kinetic model of Albery and co-workers ${ }^{17}$ resulted in an assignment of a mean rate constant of $830 \mathrm{~s}^{-1}$ and a spread parameter $\gamma$ of 0.9 .

The standard potential of the $\mathrm{FAD} / \mathrm{FADH} \mathrm{H}_{2}$ redox couple immobilized on the nanotube surface of $E^{0}=-396 \mathrm{mV}$ is very

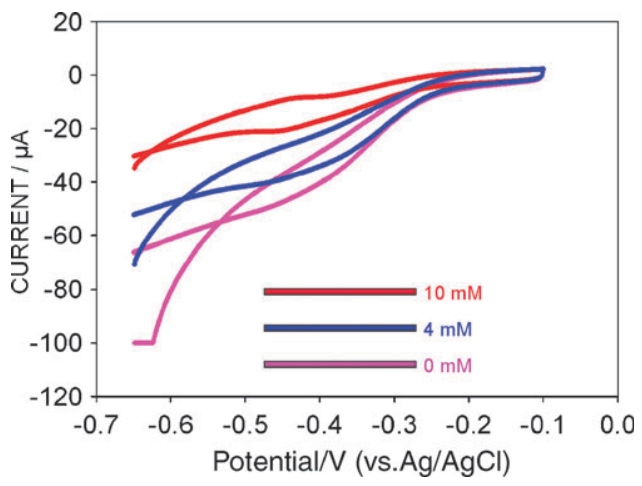

Fig. 2 Voltammetric response of SWCNT/GOx/Nafion composite electrode to glucose solutions saturated with oxygen.

close to that expected for the Flavin molecule either in aqueous solution or in the adsorbed state. It could be suggested $^{18}$ that the voltammetry presented in Fig. 1 (blue curve) corresponds to that of dissociated flavin species. We admit that FAD can adsorb on carbon electrodes. ${ }^{7}$ However we would expect that the redox behaviour in this case would be very sluggish due to the necessity of electron tunneling through a significant kinetically limiting degraded protein film barrier on the electrode surface. Correspondingly we infer from Fig. 1 that little degradation in protein structure occurs on adsorption on the SWCNT sidewall and consequently, the catalytic activity of the adsorbed enzyme molecules may remain intact. To examine the latter contention we probed the amperometric response of the SWCNT/GOx/Nafion composite modified electrode to dissolved molecular oxygen under conditions of low potential in the presence of known concentrations of glucose in the solution. In Fig. 2 the potentiodynamic response recorded for the electroreduction of molecular oxygen both in the absence and in the presence of glucose is illustrated. We note that the observed oxygen reduction current decreases when glucose is added to the solution. In Fig. 3 the difference in oxygen reduction current (recorded at a fixed potential of $-400 \mathrm{mV}$ ) between that of a glucose free solution and one containing a finite quantity of glucose is plotted as a function of glucose concentration. An excellent linear relationship is obtained between the current decrease $\Delta I$ and the glucose concentration. Hence glucose may be directly monitored in solution at an unprecedented low potential.

These observations may be understood by referring to the 'ping-pong' mechanistic scheme provided in Scheme 1 where a

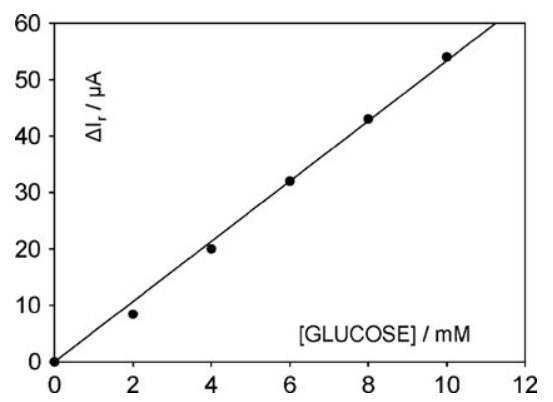

Fig. 3 Current-concentration response for oxygen reduction at a SWCNT/GOx/Nafion modified electrode in the presence of glucose. 


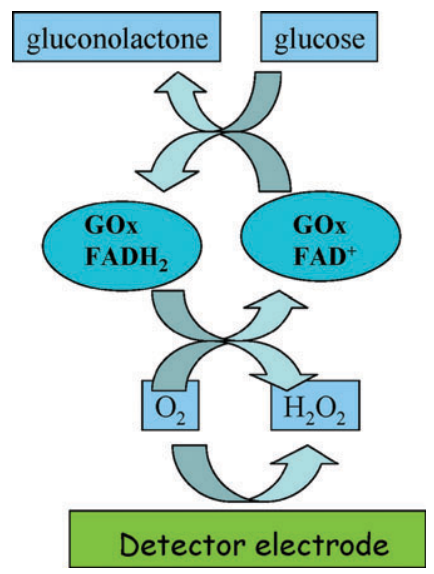

Scheme 1 Illustration of alternative pathways for molecular oxygen reduction at electrode/solution interface.

competition exists between the direct reduction of molecular oxygen at the detector electrode and the reduction of dioxygen in the reaction layer via reaction with the reduced glucose oxidase. It is clear that increasing the glucose concentration will reduce the concentration of free dioxygen at the interface and thereby result in a drop in the amperometric current response observed.

This result is significant from the perspective of the development of amperometric enzyme biosensors which will operate at low detection potential. It means that the natural cofactor for glucose oxidase may be effectively used under detection conditions where the oxidation of well known interferents such as ascorbate and uric acid does not occur. We can also infer from this result that the reduction of molecular oxygen at the dispersed SWCNT surface is relatively efficient due to the catalytic properties of the nanotube material. The question of the mechanism of catalysis remains open at the present time. It is tempting to suggest that active quinonoid groups on the nanotube surface may well enhance the electroreduction of molecular oxygen via a mediated catalytic mechanism. ${ }^{18}$ We have also shown (Fig. 4 and Fig. 5) that the composite electrode may be used at more elevated potentials, typically $0.9 \mathrm{~V}$. In this case the amperometric current response (arising from the oxidation of the hydrogen peroxide generated via oxidised enzyme regeneration, is seen to increase in

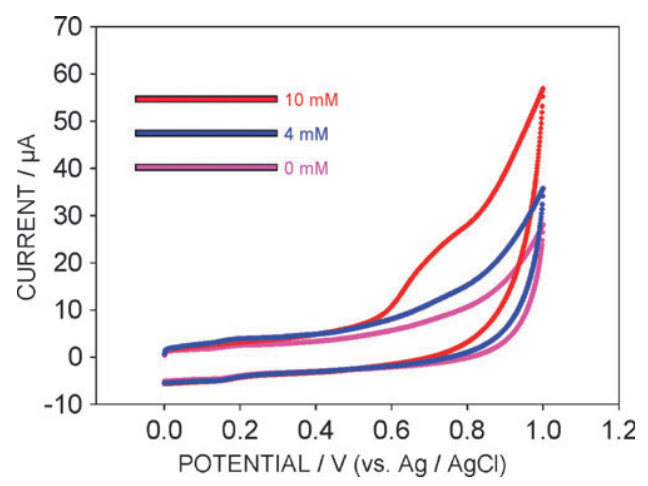

Fig. 4 Voltammetric response of SWCNT/GOx/Nafion composite electrodes in contact with oxygen saturated solution to glucose at high potential.

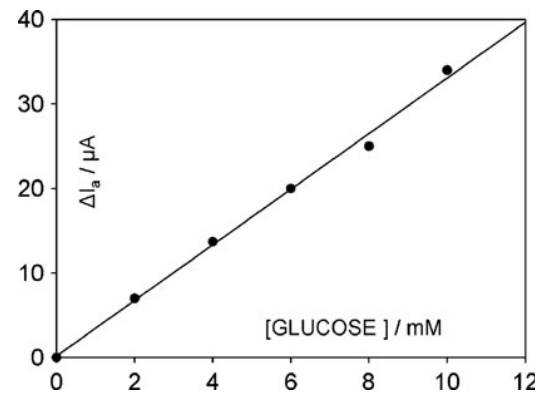

Fig. 5 Calibration curve recorded at $0.9 \mathrm{~V}$ for glucose detection at a SWCNT/GOx/Nafion composite electrode.

direct proportion to increasing glucose concentration. This detection mechanism has been established for many years, but has been shown to be prone to interference by oxidizable components in solution.

The authors are grateful for the financial support of Enterprise Ireland, Grant Number SC/2003/0049, IRCSET Grant Number SC/2002/0169 and the HEA-PRTLI Programme.

\section{Notes and references}

1 A. M. Kuznetsov and J. Ulstrup, Electron Transfer in Chemistry and Biology, Wiley, New York, 1998.

2 I. Willner, B. Willner and E. Katz, Rev. Mol. Biotechnol., 2002, 82, 325; F. A. Armstrong, Curr. Opin. Chem. Biol., 2005, 9, 110.

3 I. Willner and B. Willner, Trends Biotechnol., 2001, 19, 222; I. Willner and E. Katz, Angew. Chem., Int. Ed., 2000, 39, 1180.

4 K. Habermuller, M. Mosbach and W. Schuhmann, Fresenius' J. Anal. Chem., 2000, 366, 560.

5 W. Schuhmann, Rev. Mol. Biotechnol., 2002, 82, 425.

6 J. J. Goodring, Electrochim. Acta, 2005, 50, 3049; J. Wang, Electroanalysis, 2005, 17, 7; E. Katz and I. Willner, ChemPhysChem, 2004, 5, 1084.

7 A. Guiseppi-Elie, C. Lei and R. H. Baughman, Nanotechnology, 2002, 13, 559; C. Cai and J. Chen, Anal. Biochem., 2004, 332, 75.

8 W. Liang and Y. Zhuobin, Sensors, 2003, 3, 544; M. Wang, Y. Shen, Y. Liu, T. Wang, F. Zhao, B. Liu and S. Dong, J. Electroanal. Chem., 2005, 578, 121; Y. Yin, Y. Lu, P. Wu and C. Cai, Sensors, 2005, 5, 220.

9 G. Wang, J. J. Xu and H. Y. Chen, Electrochem. Commun., 2002, 4, 506 .

10 J. Wang and M. Musameh, Anal. Chem., 2003, 75, 2075; J. J. Goodring, R. Wibowo, J. Liu, W. Yang, D. Losie, S. Orbons, F. J. Mearns, J. G. Shapter and D. B. Hybbert, J. Am. Chem. Soc., 2003, 125, 9006; J. Liu, A. Chou, W. Rahmat, M. N. Paddon-Row and J. J. Goodring, Electroanalysis, 2005, 17, 38.

11 J. Wang, M. Musameh and Y. Lin, J. Am. Chem. Soc., 2003, 125, 2408.

12 M. J. O'Connell, P. Boul, L. M. Ericson, C. Huffman, Y. Wang, E. Haroz, C. Kuper, J. Tour, K. D. Ausman and R. E. Smalley, Chem. Phys. Lett., 2001, 342, 265.

13 C. P. Smith and H. S. White, Anal. Chem., 1992, 64, 2398; M. Ohtani, S. Kuwabata and H. Yoneyama, Anal. Chem., 1997, 69, 1045.

14 E. Laviron, J. Electroanal. Chem., 1979, 101, 19; A. P. Brown and F. C. Anson, Anal. Chem., 1977, 49, 1589; M. J. Honeychurch and G. A. Rechnitz, Electroanalysis, 1998, 10, 285; M. J. Honeychurch and G. A. Rechnitz, Electroanalysis, 1998, 10, 453.

15 I. M. Shiryava, J. P. Collman, R. Boulatov and C. J. Sunderland, Anal. Chem., 2003, 75, 494.

16 M. E. G. Lyons and G. Keeley, Sensors, 2006, 6, 1791.

17 W. J. Albery, P. N. Bartlett, C. P. Wilde and J. R. Derwent, J. Am. Chem. Soc., 1985, 107, 1854.

18 The authors note and appreciate the comments made by the referees during review concerning these items. 